\title{
Filtering Poincaré plots
}

\author{
Jaroslaw Piskorski ${ }^{1 *}$, Przemyslaw Guzik ${ }^{2}$ \\ ${ }^{1}$ Institute of Physics, University of Zielona Góra, Poland \\ ${ }^{2}$ Department of Cardiology - Intensive Therapy, University of Medical Sciences in Poznań, Poznań, Poland \\ *e-mail: jaropis@proton.if.uz.zgora.pl
}

(Rec. 22 June 2005)

\begin{abstract}
The Poincaré plot (PP) is one of the many techniques used for ascertaining the heart rate variability, which in turn is a marker of the activity of the autonomic system. Poincaré plots are very simple to produce, but their preparation involves a few fine points. This paper describes one of them, namely the filtering of data used for the Poincaré plot. We show the correct way of filtering data, present a few results of not filtering or incorrect filtering and demonstrate how proper filtering helps extract interesting information from the data. A few algorithms for preparing Poincaré plots, filtering data and calculating PP descriptors are included. As Matlab's programming language is the unquestionable standard for data analysis in the medical sciences, we illustrate these algorithms by snippets of code in this language.
\end{abstract}

Key words: computer data analysis, nonlinear dynamics, HRV, Poincaré plots

\section{INTRODUCTION}

Computers allow to analyse vast amounts of data. This apparently trivial fact is essential, however, as the use of computers as machines for analysing data gives us access to previously unavailable information. This is especially true of the medical sciences - a few dozen years ago the analysis of, for example, an electrocardiogram (ECG) was based on a recording lasting a few minutes at the most, as it is impossible for humans to analyse longer recordings. Now, the modern instrumentation, like for example the Holter recorder, allows to gather data from recordings which are 24 hours long (or longer), and the modern computer methods of data analysis allow the researcher or medical expert to derive various summary parameters, analyse recurring patterns, transform the data and much more.

The Poincaré plot (PP) is one such computer technique which makes it possible to analyse short as well as long recordings. It is both: a visual technique which can make use of the human eye's ability to recognize patterns, and a quantitative one, in the sense that it introduces various parameters (called descriptors) which quantify the information contained in a Poincaré plot $[1,2]$.

The Poincare plot is a tool developed by Henri Poincaré for analyzing complex systems. It has found its use in such diverse fields as physics and astronomy, geophysics, meteorology, mathematical biology and medical sciences [3]. In the context of medical sciences it is mainly used for quantifying the heart rate variability (HRV) and proves to be quite an effective measure of this marker $[1,2,4]$.

The PP technique summarizes the entire recording, at the same time making it possible to extract the information on short and long time behaviour of the system (heart action in this case). It is very resistant, and a reasonable Poincaré plot may be produced even from a recording containing a large amount of outliers and artifacts. In this, it is superior to, e.g., the Discrete Fourier Transform (DFT) (usually realized by the Fast Fourier Transform (FFT) algorithm), where all outliers and artifacts must be noticed and dealt with. Any failure in this respect produces an unreliable result.

So, the question arises, why Poincaré plots should be filtered at all. There are two reasons. The first of them is the obvious one: if it is possible to remove artifacts from the recording, it should be done, so as to improve the quality of the data and the Poincaré plot. The second one is perhaps more important and certainly more interesting: we filter the Poincare plot to access the information on the sinus rhythm (see Section 2), which is usually mixed with some other information. Accessing the information on sinus rhythm is important as many of the conclusions drawn from HRV analysis (like for example the one saying that $\mathrm{HRV}$ is reduced in illness and old age [4]) refer to the sinus rhythm.

The paper is organized as follows. Section 2 introduces the reader to the problem of HRV and gives background knowledge on the action of the heart. The third section introduces the Poincaré plot and the descriptors used to characterize it. In Section 4 we give general guidelines for filtering PPs and describe three filters which we routinely use in our research. Section 5 gives examples for the usefulness of the filtering procedures and describes their relation to the Poincaré plot decomposition technique [5].

In order to make this article more helpful to the reader we back our descriptions by concrete algorithms and snippets of code in a matrix programming language. Matlab is the best known matrix programming language and all the code given 
in this paper will work with this language. We use the free Matlab clone, namely Octave which can be freely downloaded from [6]. There are many more matrix languages, like Mathcad, R, Scilab, Yorick etc., and the code given in this paper should work with all these languages after introducing minor changes. Therefore, we refer to the programming language simply as the "matrix programming language", rather than giving a specific name.

The use of matrix languages simplifies all calculations involving data analysis enormously (see for example [7]), as will be clear from the examples. Very often only a line or two of code are required to solve a complex problem.

Appendix A gives some basic information on the part of the matrix language used in the present paper. Appendix B describes the data-gathering procedure.

\section{HEART RATE}

\section{AND HEART RATE VARIABILITY}

Heart beats or contractions are caused by electrical depolarization of the heart muscle [8-11]. Electrical depolarization of the different parts of heart can be observed on an electrocardiogram (ECG). The depolarization of the upper cardiac chambers, called atria (respectively right and left atrium) is visualized by the $P$-wave. The $Q, R$ and $S$ waves create the $Q R S$ complex, which represents the depolarization of cardiac lower chambers, known as ventricles (respectively right and left ventricle) (Fig. 1). The interval between successive heart beats is called $R R$ interval and it is the distance between the consecutive $Q R S$ complexes, usually measured as the distance between the $R R$ waves.

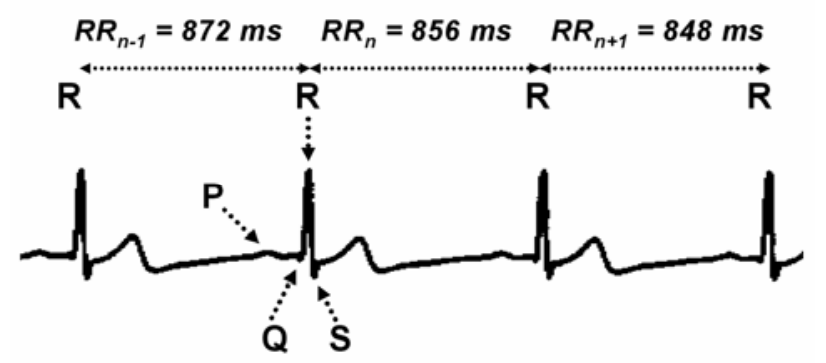

Fig. 1. A strip of ECG presenting heart's electrical activity recorded in a healthy person. The $P$ wave shows the depolarization of cardiac atria. The $Q R S$ complex characterizes depolarization of cardiac ventricles. $R$ waves of the $Q R S$ complex are always directed upward and therefore are usually used for the identification of each heart beat and its duration called the $R R$ interval (from one $R$ to the consecutive $R$ wave). In this sample there are 4 heart beats and $3 R R$ intervals between them

In healthy people the sinus node is the main rhythm generator or the primary pacemaker, with its own intrinsic activity of about $90-100$ beats per minute $[9,12]$. The excita- tory signals initiated in this node propagate through the conducting system and surrounding tissues to both atria and ventricles [11]. Physiologically, the spontaneous depolarization from the primary pacemaker is the fastest. There are, however, other pacemakers with their own spontaneous depolarizing activity. The secondary and tertiary pacemakers are localized below the sinus node and excite much slower than the primary one, which acts as a master clock $[9,11,12]$. If, for any reason, the sinus node fails to generate the primary excitation then secondary or tertiary centers with lower pacemaker frequency initiate the main depolarization wave of the heart. In healthy heart the main excitatory wave depolarizes all potential pacemakers reducing their spontaneous depolarizing activity to 0 . Thus, the excitatory signals from the sinus node prevent the generation of non-sinus beats, i.e. supraventricular (from the upper cardiac chambers) or ventricular ones (from the lower cardiac chambers). But sometimes, the supraventricular or ventricular beats appear prematurely, i.e. earlier than expected.

The momentary heart rate and the duration of the $R R$ interval is a consequence of constant interaction between the intrinsic activity of the sinus node and the influence of the autonomic nervous system, various substances circulating in the blood and present in the heart tissues $[9,10]$. Breathing appears to be the most important factor modulating heart rate $[9,10,13]$. It causes heart rate acceleration during inspiration and its deceleration during expiration. The changes in blood pressure modulated by baroreflex are another example of a separate system regulating the heart rate. The control of heart rate is modulated by both sympathetic and parasympathetic branches of autonomic nervous system as well as many other autonomic reflexes (e.g.: chemoreflexes) [10, 1315]. All these systems and reflexes are responsible for changing of the duration of $R R$ interval from one beat to another and this phenomenon is called heart rate variability (HRV) [4, 10, 13-15]. It is accepted that the higher HRV, the better prognosis in survivors of myocardial infarction or patients with heart failure. HRV is reduced in patients with diabetes and autonomic dysfunction [4].

A number of parameters are used in HRV analysis. Some of these parameters describe short-term while other depict long-term or total variability [9]. In time-domain analysis, simple statistical variables are used for HRV description. The standard deviation of all normal $R R$ intervals is one of the most popular and widely used descriptor of total HRV. In another approach, frequency-domain or spectral analysis of time series of $R R$ intervals is performed with Discrete Fourier Transformation (usually by FFT) or autoregressive models [4]. Derived from spectral HRV analysis, the total power represents total HRV, the power of high frequency (HF) corresponds to parasympathetic activity while the power of low frequency (LF) depends on both sympathetic and parasympathetic activity and their balance [4, 15]. 
However, many nonlinear phenomena are certainly involved in the genesis of HRV. It has been speculated that the analysis of HRV based on the methods of nonlinear dynamics might elicit valuable information for physiological interpretation of HRV and for the assessment of the risk of sudden death [4]. The analysis of Poincaré plots or sections of $R R$ intervals is an emerging method of nonlinear dynamics applied in HRV analysis. It appears under different names in the literature: scatter plots, first return maps, and Lorenz plots being the most prominent terms.

Other nonlinear approaches are also used for HRV analysis. A review of these methods may be found in [19].

Independently of the applied method for HRV analysis, the proper edition of ECG is always essentials. The analysis of HRV is mainly concentrated on heart beats of the sinus origin and thus all other beats (supraventricular, ventricular and paced in case of implanted artificial pacemakers) should be removed. It also happens that the raw data contain artifacts. The $R R$ intervals characteristic for incorrect beats are usually shorter (eg.: $R R i<300 \mathrm{~ms})$ or longer $(R R i>2000 \mathrm{~ms})$ than physiologically acceptable for healthy people. Another characteristics of incorrect beats may be a change of more than $20 \%$ from $R R i$ to $R R i$.

A rhythmic pattern of the heart rate may be destroyed by non-sinus beats and artifacts which usually appear too early or too late. With the use of different filters most of non-sinus beats and artifacts can be removed from the analyzed time series with $R R$ intervals. In this study we aimed to evaluate the effect of different filtering approaches on the final results of HRV analysis with the use of Poincaré plots.

\section{POINCARÉ PLOT}

We will now define the Poincaré plot for a data vector $x=\left(x_{1}, x_{2}, \ldots x_{N}\right)$ of length $N$. First, we define two auxiliary vectors:

$$
\begin{aligned}
& x^{+}=\left(x_{1}, x_{2}, \ldots, x_{N-1}\right) \\
& x^{-}=\left(x_{2}, x_{3}, \ldots, x_{N}\right) .
\end{aligned}
$$

In the medical literature these vectors are more commonly referred to as $R R i_{n}$ and $R R i_{n+1}$, respectively $[1,3,16]$.

The Poincaré plot consists of all the ordered pairs:

$$
\left(x_{i}^{+}, x_{i}^{-}\right), \quad i=1 \ldots N-1 .
$$

This may be realized in the matrix language as

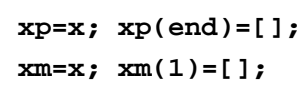

When this procedure is used for realistic data from a healthy person, we obtain a comet-like shape, similar to the one shown in Fig. 2. The Poincaré plot shown in this figure corresponds to a 4-hour recording of a healthy 23-year-old man.

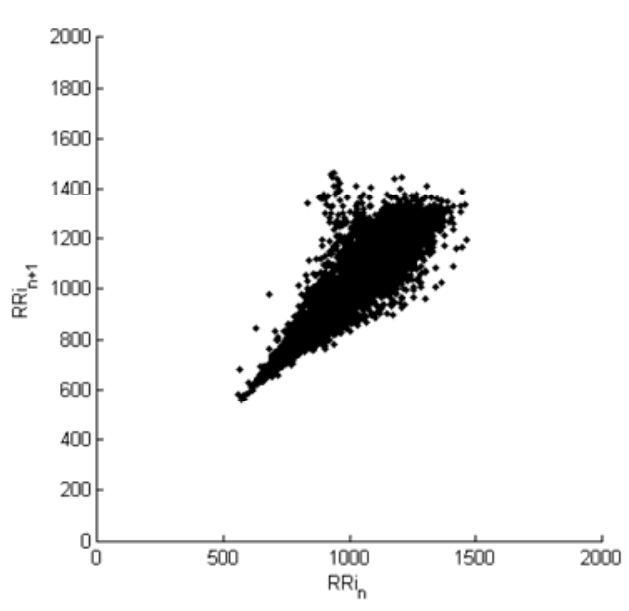

Fig. 2. The Poincaré plot of a 4-hour recording of a healthy persons

The Poincaré plot is characterized by a number of descriptors, some of which are presented in Fig. 3 [17].

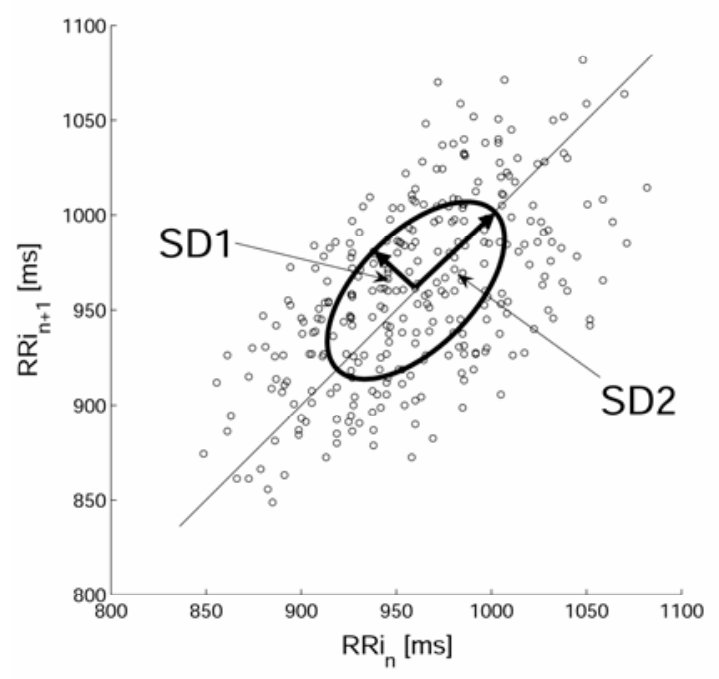

Fig. 3. Some Poincaré plot descriptors and the PP ellipse 5-minute recording (see the explanations in Section 3)

$S D 1$ and $S D 2$ are two standard Poincaré plot descriptors $[1,2]$. SD2 is defined as the standard deviation of the projection of the Poincare plot on the line of identity $(y=x)$, and $S D 1$ is the standard deviation of projection of the PP on the line perpendicular to the line of identity $(y=-x)[1]$. For our purposes we may define them as:

$$
S D 1=\sqrt{\operatorname{Var}\left(x_{1}\right)}, \quad S D 2=\sqrt{\operatorname{Var}\left(x_{2}\right)},
$$

where $\operatorname{Var}(x)$ is the variance of $x$, and

$$
x_{1}=\frac{x^{+}-x^{-}}{\sqrt{2}}, \quad x_{2}=\frac{x^{+}+x^{-}}{\sqrt{2}} .
$$


In other words, $x_{1}$ and $x_{2}$ correspond to the rotation of $x^{+}$and $\mathrm{x}^{-}$by $\theta=\pi / 4$.

$$
\left[\begin{array}{l}
x_{1} \\
x_{2}
\end{array}\right]=\left[\begin{array}{rr}
\cos \frac{\pi}{4} & -\sin \frac{\pi}{4} \\
\sin \frac{\pi}{4} & \cos \frac{\pi}{4}
\end{array}\right]\left[\begin{array}{l}
x^{+} \\
x^{-}
\end{array}\right] .
$$

This may be expressed in the matrix language as:

$\operatorname{SD} 1=\operatorname{std}(x p-x m) / \operatorname{sqrt}(2) ； \operatorname{SD} 2=\operatorname{std}(x p+x m) / \operatorname{sqrt}(2) ;$

If we define $S D R R$ as the square root of the variance of the whole time series in the recording (compare Section 2):

$$
S D R R=\sqrt{\operatorname{Var}(x)}
$$

the following approximate relation holds:
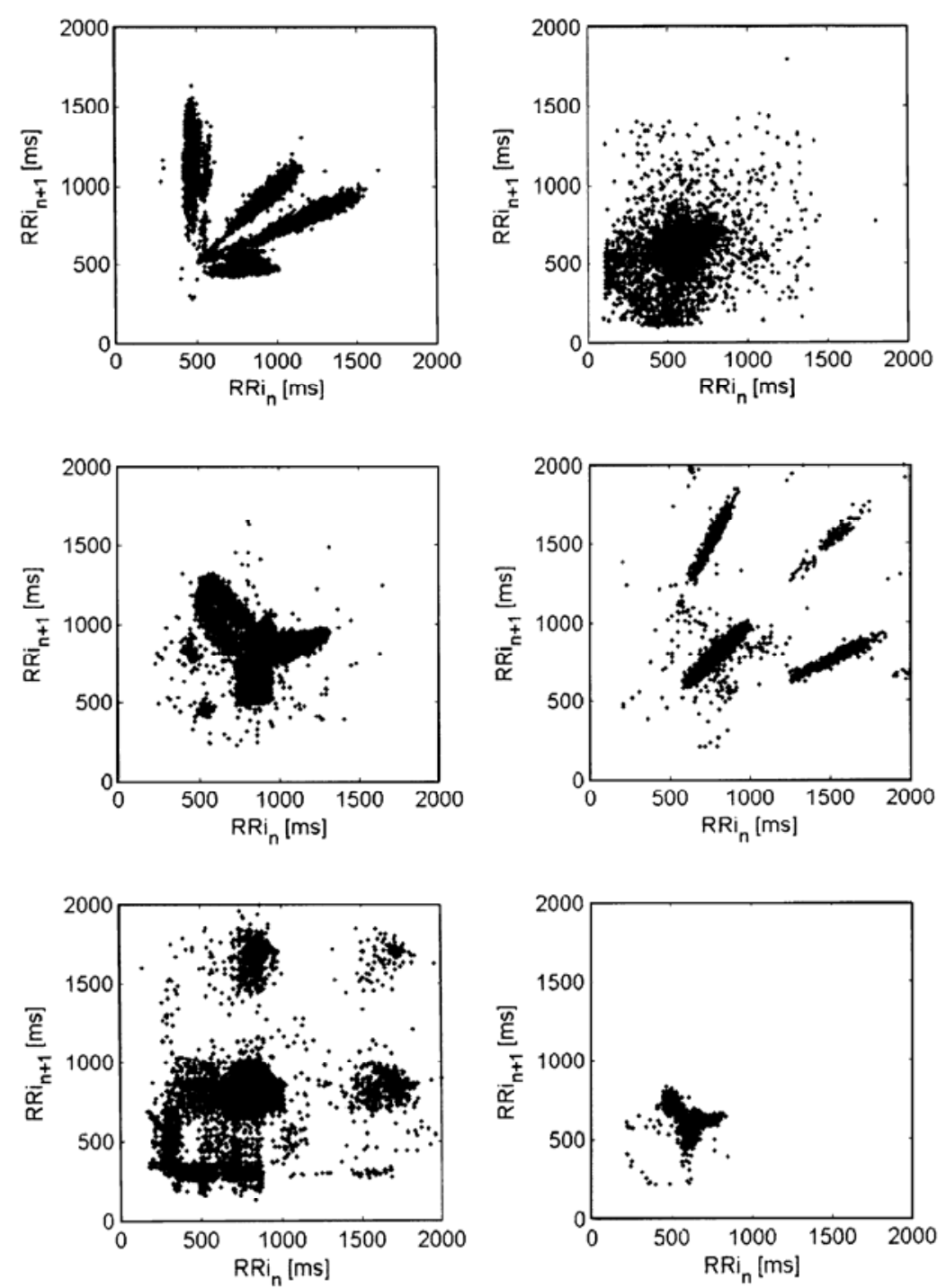

Fig. 4. Various pathological shapes of Poincaré plots - 24-hour recordings of post-MI patients with numbers of premature ventricular and supraventricular beats 
fitting takes place. This problem is known to have lead to errors made by researchers trying to actually fit an ellipse to an existing Poincaré plot. In fact the ellipse is only there to help the eye and a rectangle with sides $(2 S D 1,2 S D 2)$ might do as well.

Additionally, we may define a parameter which reflects the total variability as measured by the Poincare plot:

$$
s=\pi S D 1 S D 2
$$

which is the area of the ellipse [17, 18]. In fact the only important point is to multiply $S D 1$ and $S D 2$ - it does not have to be the area of an ellipse.

The reasons why we believe that $S$ is the total variability are given in [17]. This parameter seems to be a better measure of total variability than $S D R R$, as in the case of bigeminy, when our data vector would be similar to $x=(a, b, a, b, a, b \ldots) S D R R$ would measure a substantial variability, whereas $S$ would be equal to 0 , which agrees with the intuitive understanding of "variability".

$S D R R$ and $S$ can be calculated in the following way:

$\operatorname{SDRR}=\operatorname{std}(\mathrm{x}) ; \mathrm{S}=\mathrm{pi}$ *SD1*SD2 ;

The shape shown in Fig. 2 seems to be characteristic of healthy persons. There are, however, many other shapes, some of which have been classified as being characteristic of some pathological states ([1] and references therein). A few examples of other, pathological shapes may be found in Fig. 4.

In the next section we show how some useful information can be extracted from such plots by means of proper filtering.

\section{FILTERING POINCARÉ PLOTS}

In this section we show how to correctly remove incorrect data from the data vector. Three types of filters shall be described, together with their implementation in the matrix language. We will also demonstrate how proper filtering helps extract useful information from complex Poincaré plots.

\subsection{General notes on Poincaré plot filtering}

Let us consider a data vector similar to the one presented in Fig. 5.

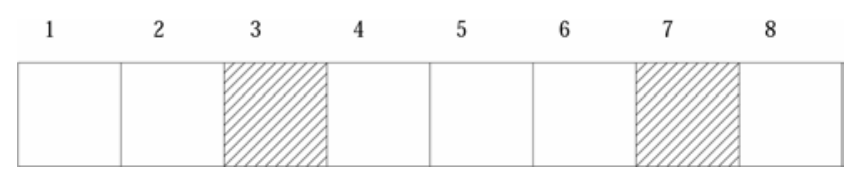

Fig. 5. An example data vector with annotated artifacts, ventricular or supraventricular beats (in this figure they are represented by filled squares)

The striped squares correspond to beats which did not originate from the sinus node. They may be artifacts, ventricular or supraventricular beats.
Very often the data gathered in a study is already annotated by the recording device, which has some built-in algorithms for recognizing incorrect beats. If this is not the case, the recognition of these beats may be carried out by specialized software. The algorithms used to do this are complex and not entirely reliable [4]. The input data for these algorithms is the full ECG rather than an $R R i$ vector.

If we want to calculate some of the parameters which describe the data vector, like for example $S D R R$ or mean $R R i$, we can simply remove the striped squares and use the remaining data vector to do this. However, if we used this vector to calculate the PP descriptors we would be making an error. The reason is illustrated in Figs 6. a-b below.

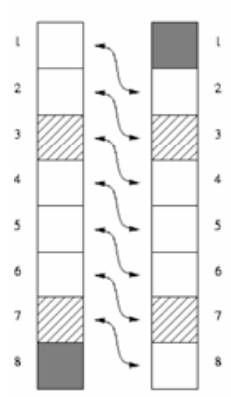

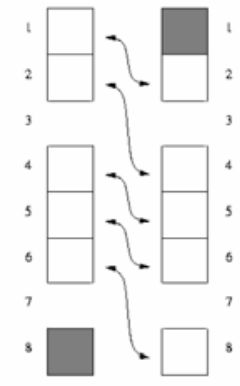

b)

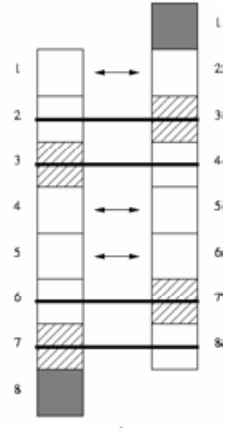

Fig. 6. The effect of filtering on the Poincaré plot. Figure a) shows a Poincaré plot without any filtering, $b$ ) is an incorrect Poincaré plot after removing artifacts, ventricular and supraventricular beats from the original data vector and c) shows the correct procedure for removing the offending beats. The filled squares correspond to the data removed while preparing the PP. The data vectors have been slightly translated in opposite directions, so that now the corresponding points are opposite one another, i.e. $(1,2),(4,5),(5,6)$

The Poincaré plot in Fig. 6 a) has been produced with the use of the original data vector, without any filtering and it will serve as a reference. In part b) of the figure the Poincaré plot has been prepared with the use of the filtered data vector, i.e. the artifacts, ventricular and supraventricular beats have been removed from the original data vector, and the resulting vector has been used to produce the plot. By comparing the result with part a) we can see, that it is incorrect as it contains some points (namely $(2,4),(6,8)$ ) which do not correspond to any real PP points and are the result of the filtering procedure only.

Part c) of the figure demonstrates the correct way of filtering the Poincaré plot. First, the Poincaré plot is prepared as described in Section 2, and only then are the incorrect beats removed from both vectors $\left(x^{+}\right.$and $\left.x^{-}\right)$, together with their counterparts in the other vector, whether the counterpart is a correct beat or not. We may lose some information in this procedure, but we can be sure that no additional information will be generated.

We will now describe three simple algorithms of filtering Poincaré plots in three cases. 


\subsection{The annotation filter}

This filter may be used with annotated recordings. Such a recording may consist of two columns: in the first one, the value of the $R R i$ is given and the second one provides the flag identifying the beat. For example in Fig. 7 we can see a fragment of a recording in which the second column contains flags 0 - for a normal beat, 1 for a ventricular beat, 2 for a supraventricular beat and 4 for an artifact.

$\begin{array}{cccccc}. . & & 1024.00 .4 . & 875.00 . & 0 \\ 945.00 . & 0 . & 1015.00 .4 . & 859.00 . & 0 \\ 930.00 . & 0 . & 1024.00 .0 . & 836.00 . & 0 \\ 961.00 . & 0 . & 1000.00 .0 . & 828.00 . & 0 \\ 969.00 . & 0 . & 984.00 .0 . & 821.00 . & 2 \\ 953.00 . & 0 . & 977.00 . & 1 . & 789.00 . & 0 \\ 937.00 . & 2 . & 945.00 .0 . & 789.00 . & 0 \\ 922.00 . & 0 . & 945.00 .0 . & 773.00 . & 0 \\ 938.00 . & 0 . & 899.00 .0 . & 930.00 . & 0 \\ 976.00 . & 0 . & 906.00 . & 2 . & . . & \end{array}$

Fig 7. An example of an annotated $R R i$-recording

Following the discussion in the previous subsection, we have to find the incorrect beats in both vectors $\left(x^{+}\right.$and $\left.x^{-}\right)$and remove them from the vector in which it was found along with its counterpart in the other vector.

This can be implemented in the matrix language as follows. First we find the indices of the incorrect beats in the original data

$L=$ length $(x)-1 ;$ indices $=f i n d(x(1: L, 2) \sim=0$;

(for details see Appendix A) and then remove the data from both vectors as follows

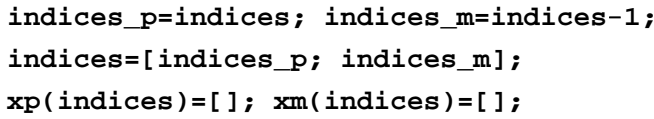

There is another point to be considered here. If the first entry should be an incorrect beat it will lead to an error, because we would be trying to remove from $x^{-}$an entry with an index 0 , which obviously does not exist. This problem isn't very difficult to solve. If a single recording is being considered, this problem can easily be solved by hand. If a large number of recordings is analysed a procedure for detecting an incorrect beat in the first position can be easily written.

\subsection{The square filter}

This filter may be used with recordings which are not annotated. We call it square because it removes data which lie outside the square $x^{ \pm}>300, x^{ \pm}<2000$. The justification for the use of this filter was given in Section 2.

The actual code in the matrix language is:

$\mathrm{L}=$ length $(\mathbf{x})-1$;

indices $=f$ ind $(x(1: L, 2)<300 \mid x(1: L, 2)>2000)$; indices_p=indices; indices_m=indices -1 ;

indices $=$ [indices_p; indices_m] ;

$\operatorname{xp}($ indices $)=[] ; \operatorname{xm}($ indices $)=[]$;

The discussion on the possible error given at the end of the previous section applies here, too.

\subsection{The quotient filter}

This filter, again, may be used with data that has not been annotated. It is the most aggressive filter, in the sense that it usually removes more points than the previous two, but at the same time it is the most prone to leave incorrect beats unfiltered. The explanation why this filter should be used can be found in Section 2.

In this filter beats fulfilling any of the following conditions:

$$
\begin{array}{lll}
\frac{x_{i}^{ \pm}}{x_{i+1}^{ \pm}} \geq 1.2, & \text { or } & \frac{x_{i}^{ \pm}}{x_{i+1}^{ \pm}} \leq 0.8, \\
\frac{x_{i+1}^{ \pm}}{x_{i}^{ \pm}} \geq 1.2, & \text { or } & \frac{x_{i+1}^{ \pm}}{x_{i}^{ \pm}} \leq 0.8
\end{array}
$$

are removed.

The code snippet for this filter is:

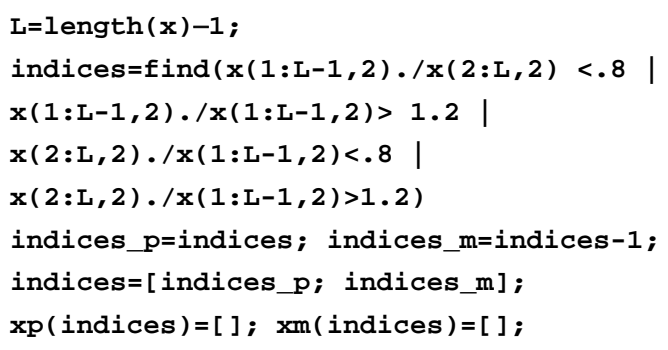

It can be easily noticed that if there are a few incorrect beats of the same order in succession, some of them will not be removed. This is why this filter works best if it is combined with any of the two other filters. Additionally, this filter can be run several times on a Poincaré plot, and each time more incorrect beats will be removed (possibly, also some correct beats will be removed in the process). This procedure of multiple application of the quotient filter has the effect of stabilizing the recording.

Again, the discussion from the end of (4.2) applies.

All of these three filters remove hardly any beats from a recording of a healthy person. However, if we consider a pathological Poincaré plot, the last filter (the quotient filter) is the most aggressive one.

\section{APPLICATIONS OF FILTERS}

In this section we will show the result of filtering pathological Poincaré plots with the filters described above. We will also describe the application of these filters for the Poincaré plot decomposition method. 


\subsection{A filtering example}

In Figure $8 \mathrm{a}, \mathrm{b}$ we can see a tachogram and a Poincaré plot of a survivor of myocardial infarction. (A tachogram is a plot of $R R$ intervals against their index in the data vector.)

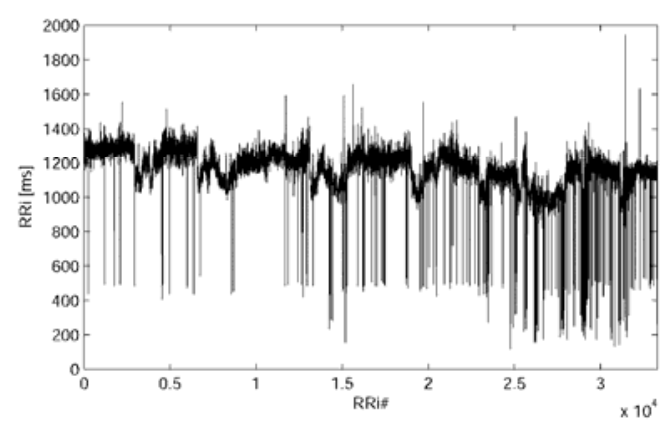

Fig. 8a. The tachogram of a non-filtered recording (12 hours)

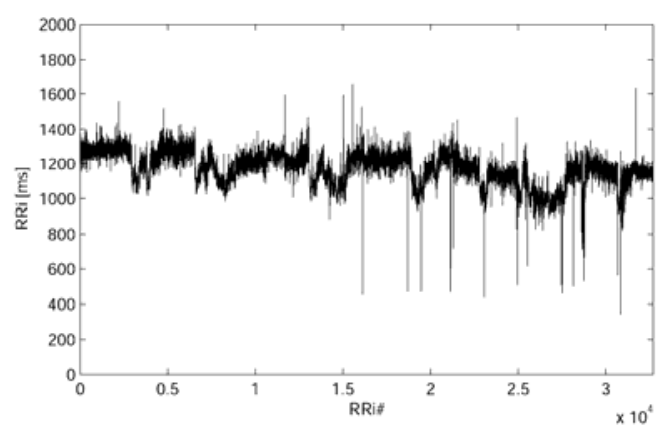

Fig. 9a. Tachogram - the same recording as in Fig. 8a after annotation filtering

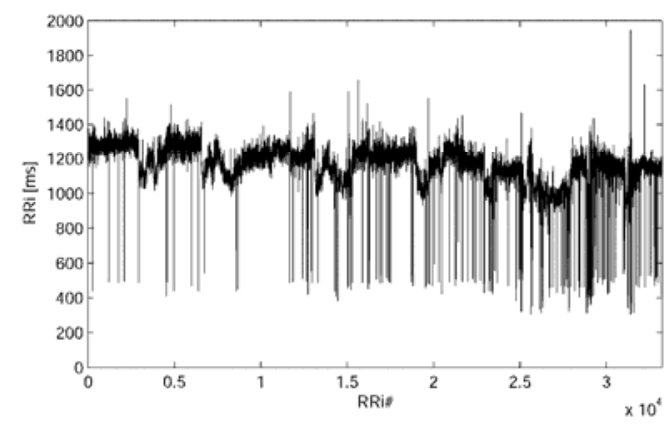

Fig. 10a. Tachogram - the same recording as in Fig. 8a after square filtering
This recording has not been filtered in any way. We can see that the Poincaré plot is very complex, and the large values of the descriptors $(S D 1=60.6 \mathrm{~ms}, S D 2=167.2 \mathrm{~ms}$ and $S=31.8 \cdot 10^{3} \mathrm{~ms}^{2}$ ) suggest a large HRV.

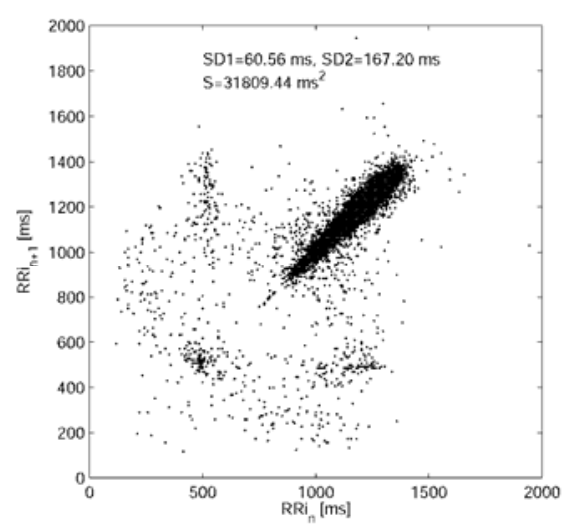

Fig. 8b. The Poincaré plot of a non-filtered recording (12 hours)

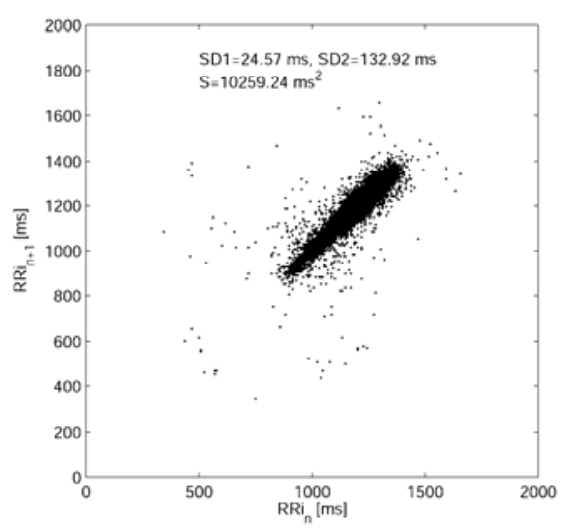

Fig. 9b. The Poincaré plot - the same recording as in Fig. 8b after annotation filtering

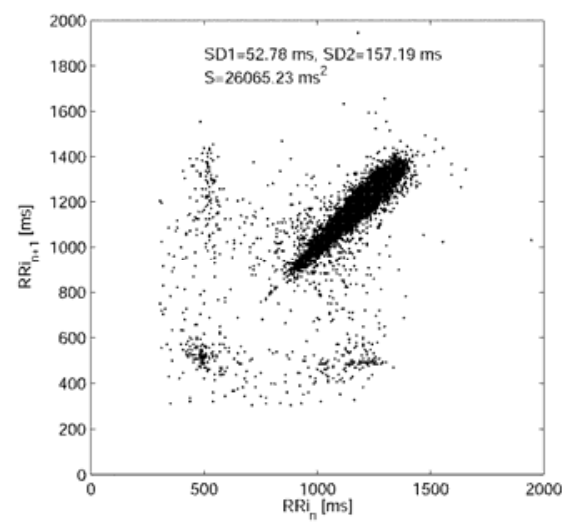

Fig. 10b. Poincaré plot - the same recording as in Fig. $8 \mathrm{~b}$ after square filtering 


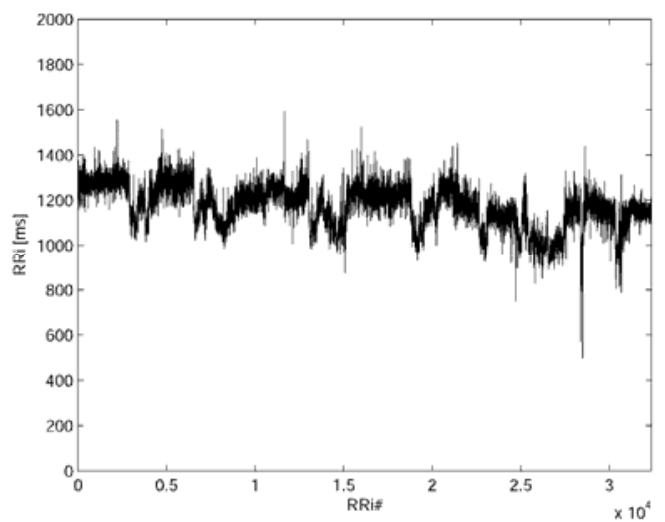

Fig. 11a. Tachogram - the same recording as in Fig. 8a after quotient filtering

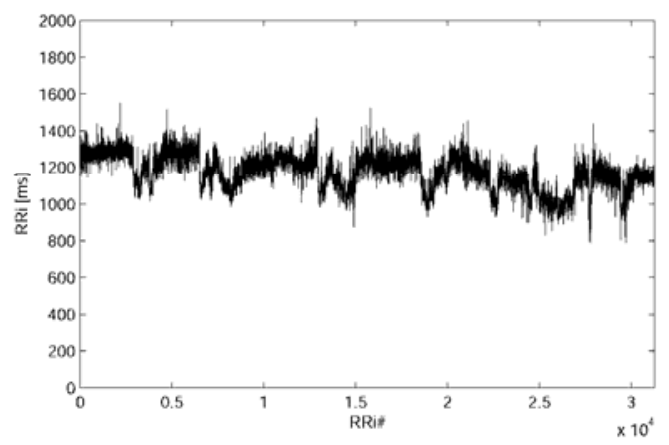

Fig. 12a. Tachogram - the same recording as in Fig. 8a after double quotient filtering

Figures $9 \mathrm{a}, \mathrm{b}$ present the same recording after annotation filtering. We can notice that the changes in the tachogram are less violent than in Figures $8 \mathrm{a}, \mathrm{b}$ but are still substantial. This may mean that the algorithm used for annotating the recording was not totally efficient. The values of the descriptors are much smaller than in the previous, unfiltered case $\left(S D 1=24.6 \mathrm{~ms}, S D 2=132.9 \mathrm{~ms}\right.$ and $\left.S=10.3 \cdot 10^{3} \mathrm{~ms}^{2}\right)$, which means that a lot of the variability found in the previous case is attributable to artifacts and ventricular and supraventricular beats.

The next figure (Fig. 10) shows the effect of the square filter applied to the same recording. The tachogram does not look very different from the unfiltered case, the descriptors are smaller $\left(S D 1=52.8 \mathrm{~ms}, S D 2=157.2 \mathrm{~ms}\right.$ and $\left.S=26.1 \cdot 10^{3} \mathrm{~ms}^{2}\right)$, but two of them are larger than in the case of the annotation filtering.

The quotient filter applied to the recording produces Fig. 11. It can be seen that the tachogram is not as chaotic as in the case of the unfiltered recording or the square filter. There are, however, some suspiciously violent jumps in the tachogram. The Poincaré plot has lost all but one of

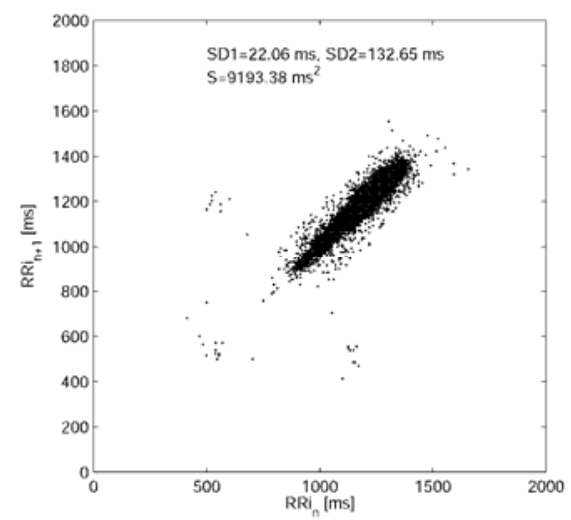

Fig. 11b. Poincaré plot - the same recording as in Fig. 8b after quotient filtering

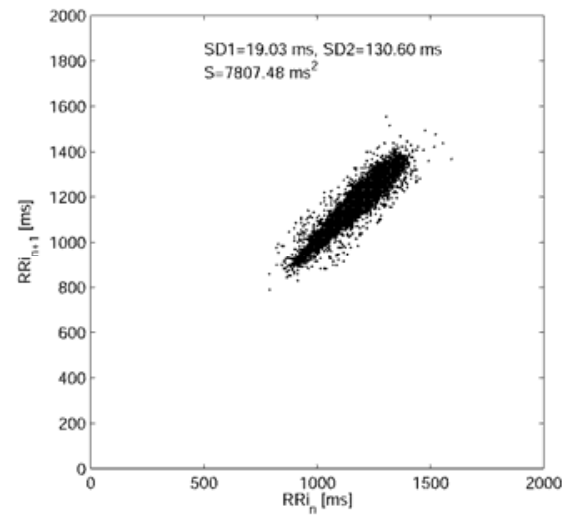

Fig. 12b. Poincaré plot - the same recording as in Fig. $8 \mathrm{~b}$ after double quotient filtering

the isolated islands visible in the previous figures. The descriptors are much smaller $(S D 1=22.1 \mathrm{~ms}, S D 2=132.7 \mathrm{~ms}$ and $S=9.2 \cdot 10^{3} \mathrm{~ms}^{2}$ ). This means that a great amount of the variability visible in the unfiltered recording is attributable to the (perhaps non-physiological) beats which fail to pass the quotient filter test.

In 4.4 it has been mentioned that the quotient filter can be run a few times on the same recording. Figure 12 presents the result of running the filter twice on the recording which we are considering here. The tachogram is now very smooth, the Poincaré plot has lost all the isolated islands and the descriptors are now very small (compared to the unfiltered case): $\left(S D 1=19.0 \mathrm{~ms}, S D 2=130.6 \mathrm{~ms}\right.$ and $\left.S=7.8 \cdot 10^{3} \mathrm{~ms}^{2}\right)$. For reasons given in the Introduction and Section 2 we believe that this tachogram and Poincaré plot correspond to the sinus node only.

In the next section we will see even more clearly that the assumption that the filtered recordings correspond to the sinus node agrees with the assumption of diminished HRV in illness. 


\subsection{Poincaré plot filtering in the decomposition technique}

The Poincaré plot decomposition [5] is a visual technique for assessing the HRV. The reader may want to download the movies demonstrating this technique form [20] to better understand further explanations.

The recording is analyzed with the use of a sliding window which is 5 minutes long and moves smoothly along the tachogram - in the tachogram this window is marked by a red area. Every second the sliding window subtends a 5-minute long recording, and for each position of the window a Poincaré plot is prepared and all the descriptors calculated. Each frame of the movie corresponds to a position of the sliding window.

Movie $1 \mathrm{u}$ contains a recording of a healthy person - we can observe how the Poincaré plot fans out for large $R R$ intervals and is compressed for small $R R$ intervals. The second panel presents the movement of the point $(S D 1(t), S D 2(t))$ in the ( $S D 1, S D 2)$ space. The movement is mostly vertical. This recording is not filtered, and there is no movie for the filtered recording, as it looks basically the same.

Movie $2 \mathrm{u}$ contains a unfiltered recording of a survivor of myocardial infarction. We can see that the tachogram and the Poincare plot are similar to what we have seen in the previous subsection. The movements of the point in the second panel are no longer smooth or mostly vertical.

When this recording is filtered with a combination of the annotation filter and the quotient filter - in movie $2 \mathrm{f}-$ the situation is totally different. The variability is much smaller than in the unfiltered recording and possibly smaller than in the case of the healthy person, which can be noticed by observing the instantaneous Poincaré plot, which consists of the red points. The movement of the point in the second panel is much more confined and spans a smaller area.

The next movie, $3 \mathrm{u}$, looks very different. The shape of the Poincaré plot is very complex and the $(S D 1(t), S D 2(t))$ point moves in a very chaotic way. If this recording were to be the basis for assessing HRV, the conclusion would have to be that the variability is large.

However, when this recording is filtered (movie $3 \mathrm{f}$ ), again with a combination of the annotation and quotient filters, we can see almost no variability! The Poincaré plot is a small circular patch which hardly moves and the $(S D 1(t), S D 2(t))$ point spans a very small area. The person, whose recording can be seen in movies $3 \mathrm{u}$ and $3 \mathrm{f}$ died shortly after the recording.

The person whose condition was the worst had the smallest HRV, but this could only be observed after the proper filtering of the recording.

\section{CONCLUSIONS}

In the paper we have introduced the reader to the problem of measuring HRV with the use of filtered Poincaré plot. We have described the general guidelines for filtering and given details of three different filters, namely the annotation filter, the square filter and the quotient filter. In Section 5 we have shown that the filters extract useful information from Poincaré plots, and that the descriptors become good measures of HRV only after the application of the filters. Indeed, the big values of descriptors indicating large values of HRV become small after proper filtering. There are reasons to believe, that the filtered recordings contain information on the sinus node.

Each technique has been illustrated by a snippet of code in the matrix language.

The Poincare plot is an incredibly simple technique for measuring HRV. Additionally, the filtering procedures pose no problem either. If we compare the Poincaré plot and the Discrete Fourier Transform we will notice that in some respects the PP is superior to the DFT - and filtering is one of them. Filtering data for DFT (FFT) is very difficult and in fact creates and/or changes the information contained in the recording.

The Poincaré plot is a new and promising technique of HRV analysis. It is so simple, that it can be used by anyone with a computer and a basic knowledge of programming (preferably in a matrix language). However, any Poincaré plot analysis of a real data vector should be preceded by a careful look at the data and the application of the suitable filtering procedure.

\section{Appendix A: Basics of the matrix language}

In this Appendix we will briefly describe the techniques used in the present paper.

$\mathbf{x}-$ is a matrix or a vector defined as $\mathbf{x}=[\mathbf{a}, \mathbf{b}, \mathbf{c}, \ldots]$,

$\mathbf{x}(\mathbf{i})-$ is the $i$-th element of matrix $\mathbf{x}$, treated here as a vector,

$\mathbf{x}(i, j)-$ is the $x_{i, j}$ element of the matrix,

$\mathbf{x}(:, 1)-$ is the first column of the matrix,

$\mathbf{x}(1,:)-$ is the first row of the matrix,

find $(x>a)$ - returns a vector of indices of the elements of $\mathbf{x}$ which satisfy the condition $x_{i, j}>a$,

$==$, $<, ~ \sim, \mid-$ are the logical expressions: equals, is smaller / greater, not, or, respectively,

.$/$ - is the element-wise division,

$\mathbf{x}([\mathbf{i}, \mathbf{j}, \mathbf{k}, \mathbf{m}, \mathbf{n}])=[]-\operatorname{removes} x_{i}, x_{j}, x_{k}, x_{m}, x_{n}$ from the data vector $\mathbf{x}$.

\section{Appendix B: Data gathering}

This Appendix describes the data gathering process for the datasets used in this paper.

The recordings were visually inspected with the use of professional Holter ECG recording and analyzing system (Zymed 1810, Philips, USA). All necessary corrections of beats with their proper identification was done basing on the preliminary automatic evaluation and then manually changed if necessary in some cases. After the proper identification of all heart beats, the collected recordings were exported into an 
ASCII file containing the duration of all $R R$ intervals with their labeling. Number of $R R$ intervals in the analyzed ASCII files was in the range of approximately 60,000 to 140,000 . The following abbreviations were used for the labeling: 0 for normal beats of sinus origin, 1 for supraventricular beats, 2 for ventricular beats and 4 for artifacts. As the sampling frequency of the used recording system is $200 \mathrm{~Hz}$ therefore the precision of $R R$ interval identification is $5 \mathrm{~ms}$.

\section{References}

[1] M. Brennan, M. Palaniswami and P. Kamen, IEEE Trans Biomed. Eng. 48, 1342 (2001)

[2] M. Brennan, M. Palaniswami and P. Kamen, Am. J. Physiol. Heart Circ. Physiol. 283, H1873 (2002).

[3] E. Ott, Chaos in dynamical systems (Cambridge University Press, Cambridge 1993).

[4] Heart rate variability. Standards o measurement, physiological interpretation, and clinical use. Task Force of the Working Groups on Arrhythmias and Computers in Cardiology of the ESC and the North American Society of Pacing and Electrophysiology (NASPE), European Heart Journal 93 , 1043-1065 (1996)

[5] P. Guzik and J. Piskorski, Folia Cardiologica 12 suppl. D, O271:432-435 (2005).

[6] The interpreter of the Octave language may be downloaded from www.octave.org

[7] S. T. Karris, Signals and systems with Matlab applications (Orchard Publications, Fermont, 2003).

[8] P. F. Carleton, Physiology of the cardiovascular system, in: Pathophysiology. Clinical aspects of disease process edited by S. A. Price (Mosby Year Book, St. Louis 1992) pp. 382-392.
[9] W. Wiedmeier, Cardiac and circulatory physiology in: Essential of human physiology. Integrated Medical Curriculum edited by T. M. Nosek (1999); chapter 3: http://imc.gsm.com

[10] R. Hainsworth, Physiology of the cardiac autonomic system in: Clinical guide to cardiac autonomic tests edited by M. Malik (Kluwer Academic Publishers, London 1998) pp. 3-28.

[11] B. Surawicz and T. K. Knilans, Normal electrocardiogram: origin and description in Chou's electrocardiography in clinical practice. Adult and pediatric edited by B. Surawicz, T. K. Knilans (W. B. Saunders Company. New York 2001) pp. 3-27.

[12] R. Mrowka, P. B. Persson, H. Theres and A. Patzak, Am. J. Physiol. Regul. Integr. Comp. Physiol. 279, R1171 (2000).

[13] D. L. Eckberg, J. Physiol. 548, 339 (2003).

[14] G. Parati, J. P. Saul and P. Castiglioni, J. Hypertens 22, 1259 (2004).

[15] D. L. Eckberg, Circulation 96, 3224 (1997).

[16] M. R. Guevara, L. Glass and A. Shrire, Science 214, 1350 (1981).

[17] P. Guzik, J. Piskorski, T. Krauze, R. Schneider, K. H. Wesseling, A. Wykrętowicz, H. Wysocki, Folia Cardiologica 12, suppl. D, P013:17-20 (2005).

[18] P. Guzik, J. Piskorski, B. Bychowiec, A. Węgrzynowski, T. Krauze, R. Schneider, P. Liszkowski, A, Wykrętowicz, B. Wierusz-Wysocka and H. Wysocki, Folia Cardiologica 12, suppl. D, P030:64-67 (2005).

[19] A. J. E. Seely, P. T. Macklem, Critical Care 6, R367 (2004).

[20] The movies can be downloaded from www.if.uz.zgora.pl/ $\sim$ jaropis/filters.html

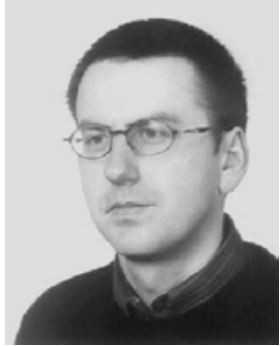

Dr. JAROSŁaw PISKORSKI works at the Institute of Physics of the University of Zielona Gora, Poland. His main areas of interest include elementary particle physics (mainly discrete symmetries and their violation) and the application of the methods of statistical physics and nonlinear dynamics to heart rate variability.

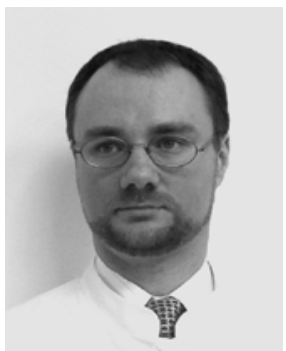

Dr. Przemyslaw GuziK is both a medical doctor and scientist working at the Department of Cardiology - Intensive Therapy, University of Medical Sciences in Poznan, Poland. Non-invasive evaluation of cardiovascular system and autonomic modulation of heart rate belong to his research topic. The analysis of various cardiovascular time series (heart rate, blood pressure) allow him to focus on heart rate variability, heart rate turbulence, baroreflex sensitivity and arterial stiffness. The use of Poincare plots of heart rate, which is his latest area of interest and research, is one of the approaches used to investigate heart rate variability. 